\title{
Scaling solutions in general non-minimal coupling theories
}

\author{
Luca Amendola \\ Osservatorio Astronomico di Roma, Viale del Parco Mellini 84 \\ 00136 Roma, Italy \\ (February 1, 2008)
}

\begin{abstract}
A class of generalized non-minimal coupling theories is investigated, in search of scaling attractors able to provide an accelerated expansion at the present time. Solutions are found in the strong coupling regime and when the coupling function and the potential verify a simple relation. In such cases, which include power law and exponential functions, the dynamics is independent of the exact form of the coupling and the potential. The constraint from the time variability of $G$, however, limits the fraction of energy in the scalar field to less than $4 \%$ of the total energy density, and excludes accelerated solutions at the present.
\end{abstract}

\section{INTRODUCTION}

A great deal of effort has been devoted in recent times to the dynamics of scalar fields in the radiation and matter dominated era. The motivations are manyfold: first, several theories of fundamental physics predict the existence of scalar fields [1] [2] [3]; second, a slowly rolling scalar field may mimic the behavior of a cosmological constant at the present time, in agreement with a popular model of structure formation and with the observation of an accelerated space expansion [1] [4] [5]; third, the scalar field may alleviate the constraints on a true cosmological constant [6]; fourth, the additional source of fluctuations produced by the scalar field may give new observable effects on the cosmic microwave background and on the structure formation [2] [7] [8].

So far, most work focused on fields with minimal coupling to gravity [1] [2] [4] [5] [9] [10]. In this case, the dynamics in a homogeneous and isotropic space-time is completely determined once one specifies the matter fluid equation of state and the field potential. For the former, the obvious choices of interest are the equation of state of a relativistic fluid and of a pressureless one. For the latter, although there are no observations or fundamental principles to guide our investigation, potentials like power-laws, exponential and a handful of other cases have been selected, basing either on simplicity or on some particle physics model. Among the infinite solutions of the system of equations, the attractor solutions are of course of the greatest interest. Among the attractors, those which have a power-law behavior, denoted also as scaling solutions, are particularly simple to find and to study. Consequently, the study of the scalar field dynamics has focussed on the search of scaling attractors. To be interesting for cosmological purposes, these attractor solutions must also lead to a energy density in the scalar field which is a non-negligible fraction of the total energy density. Finally, if we want to explain recent observations of the large-scale geometry of the space-time [11] [12], the scalar factor has to be accelerated at the present.

In minimal coupling theories the Lagrangian is the sum of the Einstein-Hilbert gravity Lagrangian and of the scalar field sector. The non-minimal coupling (NMC) adds a new term which, in its simplest form, may be written as (for a more general form that includes derivatives see Ref. [13])

$$
f(\phi) R \text {. }
$$

For instance, Refs. [14] [15] [16] [17] adopted $f \sim \phi^{2}$ discussing the model in the context of inflation. In Ref. [18] several attractor scaling solutions in the matter dominated regime with power-law and exponential potential have been found. Other forms of $f(\phi)$ have been considered (see e.g. [19]).

A common feature of all these investigations, perhaps obviously, is the choice of specific potentials and coupling functions. The purpose of this paper is to show that it is possible to find attractor solutions in NMC models in which both the coupling $f(\phi)$ and the potential $V(\phi)$ are left unspecified, and only their relation matters. In other words, we will find a class of models in which the dynamics of the system is independent of the coupling and of the potential, and depends only on their relation. In particular, we will find attractor solutions for all models for which we can write 20]

$$
V(\phi)=A f(\phi)^{M} .
$$

This relation holds, for instance, when both $V$ and $f$ are power-law, or exponential, but is also valid for much more complicated functions, like products of power law and exponential. In the limit of strong coupling, the dynamics of the cosmological solution will be shown to depend essentially only on $M$ and on the fluid matter equation of state. 
After performing a conformal rescaling of the metric, the NMC system is written as a scalar field in pure General Relativity with an exponential potential and an extra coupling to the ordinary matter (see e.g. [21], in which however only the case $f \sim \phi^{2}$ has been discussed). This system shows a surprisingly rich phase space structure, with four different attractors. Two of these are qualitatively similar to the attractors found in the system without extra coupling. The other two however are new, and have not been previously identified. Although we derived this system from a class of NMC theories, we remark that it is interesting on its own, and many cosmological properties of its trajectories have yet to be worked out. Here we study it mainly to constrain the NMC model, and find that the constraint on the variability of the gravitational constant rules out this class of models as explanation for the accelerated expansion rate of the Universe.

In the next section we work out the field equations. In Section 3 we find and discuss the attractor solutions, in Section 4 we discuss their cosmological properties, and in the final Section we draw the conclusion and point to new developments.

\section{FIELD EQUATIONS}

Consider the Lagrangian of a NMC scalar field plus a perfect fluid matter component $\left(\kappa^{2} \equiv 8 \pi M_{p}^{-2}\right)$

$$
\begin{aligned}
L_{\text {tot }} & =L(\phi, R)+2 \kappa^{2} L_{\phi}+2 \kappa^{2} L_{\text {matter }}, \\
L(\phi, R) & =-f(\phi) R \\
L_{\phi} & =\frac{1}{2} \phi_{, \mu} \phi^{, \mu}-V(\phi) .
\end{aligned}
$$

Contrary to the usual notation, we found convenient to include into $f(\phi)$ the constant that produces the Einstein-Hilbert term, so that our $f$ is $1+\kappa^{2} \xi f(\phi)$ in the notation of, e.g., Ref. [14 [15] [16] [17]). We will always assume $f>0$, since it acts as an effective gravitational constant,

$$
G_{e f f}=\left(2 \kappa^{2} f\right)^{-1}
$$

The Einstein equations are

$$
G_{\mu \nu}=L_{, R}^{-1}\left[\frac{1}{2} g_{\mu \nu}\left(L-L_{, R} R\right)-g_{\mu \nu} \square L_{, R}+\left(L_{, R}\right)_{; \mu \nu}+\kappa^{2} T_{\mu \nu(\phi)}+\kappa^{2} T_{\mu \nu(m)}\right]
$$

where $L_{, R}$ denotes here $d L / d R$, and where the scalar field energy-momentum tensor is

$$
T_{\mu \nu(\phi)}=\phi_{, \mu} \phi_{, \nu}-\frac{1}{2} g_{\mu v} \phi_{, \alpha} \phi^{, \alpha}+g_{\mu v} V(\phi),
$$

and the fluid tensor is

$$
T_{\mu \nu(m)}=(\rho+p) u_{\mu} u_{\nu}-g_{\mu \nu} p
$$

Now, under the conformal transformation

$$
\widetilde{g}_{\mu v}=e^{2 \omega} g_{\mu v},
$$

the following transformations (see e .g. [23] 24] [25]) occur: the kinetic term

$$
K_{\mu \nu(\phi)}=\phi_{, \mu} \phi_{, \nu}-\frac{1}{2} g_{\mu v} \phi_{, \alpha} \phi^{, \alpha}
$$

remains invaried $\left(K_{\mu \nu}=\widetilde{K}_{\mu \nu}\right)$; the potential term $g_{\mu v} V(\phi)$ becomes $e^{-2 \omega} \widetilde{g}_{\mu v} V(\phi)$; and the perfect fluid tensor becomes

$$
T_{\mu \nu(m)}=e^{-2 \omega} \widetilde{T}_{\mu \nu(m)}
$$

Putting

$$
2 \omega=\log f,
$$

it follows that the equations in the rescaled metric (sometimes called Einstein frame, while the old metric is the Jordan frame) are 


$$
\widetilde{G}_{\mu \nu}=\kappa^{2}\left[F^{2}(\phi) \widetilde{K}_{\mu \nu(\phi)}+\widetilde{g}_{\mu \nu} e^{-4 \omega} V(\phi)+e^{-4 \omega} \widetilde{T}_{\mu \nu(m)}\right]
$$

where

$$
F^{2}(\phi)=\frac{1}{f}+\left(\frac{f^{\prime}}{c f}\right)^{2},
$$

where $c^{2}=2 \kappa^{2} / 3$ and where the prime denotes derivation with respect to $\phi$. We can then define a new canonical field

$$
\psi \equiv \int d \phi F(\phi)
$$

a new potential

$$
U(\psi) \equiv \frac{V(\phi)}{f(\phi)^{2}}
$$

and a new matter tensor

$$
\widetilde{T}_{\mu \nu(m)}^{*} \equiv e^{-4 \omega} \widetilde{T}_{\mu \nu(m)} .
$$

Finally, all these definitions lead to the canonical equations in the new metric $\widetilde{g}_{\mu \nu}$

$$
\widetilde{G}_{\mu \nu}=\kappa^{2}\left[\widetilde{T}_{\mu \nu(\psi)}+\widetilde{T}_{\mu \nu(m)}^{*}\right] .
$$

The new matter energy-momentum tensor can be written as

$$
\widetilde{T}_{\mu(m)}^{* \nu}=\operatorname{diag}\left(\rho e^{-4 \omega},-p e^{-4 \omega},-p e^{-4 \omega},-p e^{-4 \omega}\right)=\operatorname{diag}\left(\rho^{*},-p^{*},-p^{*},-p^{*}\right) .
$$

As a last step, we rewrite the new metric in the Friedmannian form

$$
\widetilde{g}_{\mu \nu}=\operatorname{diag}\left(1,-\widetilde{a}^{2}-\widetilde{a}^{2},-\widetilde{a}^{2}\right),
$$

where the old time and the old scale factor are

$$
t=\int e^{-\omega(\tilde{t})} d \widetilde{t}
$$

and

$$
a=e^{-\omega(\widetilde{t})} \widetilde{a} .
$$

The equation of motion for the fields are obtained as the covariant conservation laws of the energy tensors. In the old frame they read

$$
\begin{aligned}
\square \phi+V^{\prime}+f^{\prime} R / 2 \kappa^{2} & =0, \\
T_{\mu \nu(\phi)}^{; \mu} & =0 .
\end{aligned}
$$

The transformation to the new frame is performed according to the rules

$$
\begin{aligned}
& R=e^{2 \omega}\left(\widetilde{R}-6 \widetilde{g}^{\alpha \beta} \omega_{, \alpha} \omega_{, \beta}+6 \square \omega\right), \\
& \square=e^{2 \omega}\left(\square-2 \widetilde{g}^{\alpha \beta} \omega_{, \alpha} \nabla_{\beta}\right) .
\end{aligned}
$$

From now on, we omit all the tilde, until we return to the original quantities. Finally, the full set of equations in the Friedmann metric read:

$$
\begin{aligned}
\ddot{\psi}+3 H \dot{\psi}+U_{, \psi} & =\frac{1}{2} W_{, \psi}\left(\rho^{*}-3 p^{*}\right), \\
\dot{\rho}^{*}+3 H\left(\rho^{*}+p^{*}\right) & =-\frac{1}{2} W_{, \psi} \dot{\psi}\left(\rho^{*}-3 p^{*}\right), \\
3 H^{2} & =\kappa^{2}\left(\rho^{*}+\frac{1}{2} \dot{\psi}^{2}+U\right), \\
-2 \dot{H} & =\kappa^{2}\left(\rho^{*}+p^{*}+\dot{\psi}^{2}\right),
\end{aligned}
$$


where

$$
W=\log f(\phi), \quad W_{, \psi}=\frac{f^{\prime}}{f F} .
$$

As already remarked in the Introduction, the system (26-29), here derived from a NMC model, is interesting on its own. Indeed, we can regard either the Jordan or the Einstein frame as the physical one. In the former case, we have to express the solutions of the above system back in the original frame, and study its cosmological consequences in the original frame, as we will do below. In the latter case, the solutions of the system are the physical solutions, and their properties can be directly compared to observations. In particular, the constraints from the variability of $G$, which we will find to limit heavily the cosmological viability of our solutions, apply only assuming the physical frame to be the original Jordan one.

\section{SOLUTIONS}

The full dynamics of the system $(26-29)$ is specified by the potential $U$ and by the equation of state $p=(w-1) \rho$. In the following we consider only $0 \leq w \leq 2$. To write down the potential $U(\psi)$, we have first to find the relation between $\psi$ and $\phi$. This is where the possibility of a dynamics independent of the potential and of the coupling function arises. In fact, if we assume that 20]

$$
f^{\prime 2} \gg c^{2} f
$$

then we can simplify Eq. (15):

$$
F^{2}(\phi)=\left(\frac{f^{\prime}}{c f}\right)^{2}
$$

It follows

$$
c \psi=\int \frac{d f}{f}=\log f
$$

where the integration constant can be absorbed into a redefinition of $\psi$. It follows that the conformal function $\omega$ equals $c \psi / 2$. Therefore, once we have the dynamics of $\psi$ in the transformed metric, we can write down the solution in terms of the original metric without having to specify $f(\phi)$, provided we express also the potential $V(\phi)$ as a function of $f(\phi)$. With the assumption (31) we get $W_{, \psi}=c$ in the system (26 29), so that putting

$$
\beta=4-3 w
$$

the first two equations become

$$
\begin{aligned}
\ddot{\psi}+3 H \dot{\psi}+U_{, \psi} & =\frac{1}{2} c \beta \rho^{*}, \\
\dot{\rho}^{*}+3 H w \rho^{*} & =-\frac{1}{2} c \beta \dot{\psi} \rho^{*} .
\end{aligned}
$$

The condition (31) holds true in several cases. For instance, it is verified for large $\phi$ by any function $f(\phi)$ which grows faster than quadratically, that is $\lim _{\phi \rightarrow \infty} f(\phi) / \phi^{2} \rightarrow \infty$. In the often-studied quadratic case, $f=1+\kappa^{2} \xi \phi^{2}$, for large $\phi$ we can put $f(\phi)=\kappa^{2} \xi \phi^{2}$. Then, instead of Eq. (31), one has $f^{\prime 2}=4 \kappa^{2} \xi f$ , and all that changes is that in Eq. (32) and Eq. (33) $c^{2}$ is replaced by $c^{2} /(1+1 / 6 \xi)$. In this case, all the results found below become exact. The weak coupling limit in which $\kappa^{2} \xi \phi^{2} \ll 1$, i.e. $\xi \ll\left(\kappa^{2} \phi^{2}\right)^{-1}$, on the other hand, is excluded in the present analysis. We could then label our case as the strong coupling limit. In fact, it is easily seen that it corresponds to the limit in which the Lagrangian can be approximated as $-f(\phi) R-2 \kappa^{2} V(\phi)$, neglecting the kinetic term $\frac{1}{2} \phi_{, \mu} \phi^{, \mu}$. Notice however that this does not imply that the scalar field kinetic terms in the field equations are negligible, because the non-minimal coupling itself introduces other kinetic terms.

Now, as anticipated, suppose we can write $V(\phi)=A f(\phi)^{M}$. The potential becomes then

$$
U(\psi)=\frac{A f(\phi)^{M}}{f(\phi)^{2}}=A e^{\sqrt{2 / 3} \mu \kappa \psi},
$$

where 


$$
\mu \equiv M-2 .
$$

Therefore, the potential can be written as an exponential, whatever the shape of $V$ and of $f$, provided that the condition (31) and the relation (2) are fulfilled. The sign of $\mu$ selects the direction in which the field $\psi$, and thus the variable $f$, rolls. If $\mu>0, \psi$ rolls toward $-\infty$, so that $f \rightarrow 0$, and the effective gravitational constant $G_{e f f}$ increases with time. In the opposite case, $\mu<0$, we have that $G_{e f f}$ decreases in the future. We emphasize that if $f(\phi)$ is quadratic, then all results below remain valid provided $\mu$ is replaced by $\mu_{q}=\mu /(1+1 / 6 \xi)^{1 / 2}$ and $\beta$ by $\beta_{q}=\beta /(1+1 / 6 \xi)^{1 / 2}$.

The scalar field dynamics in NMC theories is then reduced to the scalar field dynamics in pure general relativity with an exponential potential and with a scalar field/matter coupling. In the radiation case in which $w=4 / 3$, we have $\beta=0$, and the source terms decouple. The decoupling occurs also when we can neglect the matter energy density $\Omega_{\rho^{*}}=\kappa^{2} \rho^{*} / 3 H^{2}$ with respect to the scalar field energy density $\Omega_{\psi}$. In these cases, the problem is identical to that already solved in, e.g., Ref. [2] [9] [10] [22]. The case $\beta \neq 0$ has been already discussed by Wetterich in [21], where some of its attractors have been identified. Here we extend the analysis to the full classification of critical points and attractors (finding two new attractors) and express the solutions in terms of the old frame. We keep $\beta$ as an independent parameter as long as possible, and proceed to replacing it by $4-3 w$ only in the graphics, in order to narrow the parameter space to two dimensions, namely $w$ and $\mu$. The formulas apply however to the more general case, unless otherwise specified.

Following Copeland et al. [10] we define

$$
x=\frac{\kappa \dot{\psi}}{\sqrt{6} H}, \quad y=\frac{\kappa \sqrt{U}}{\sqrt{3} H},
$$

and introduce the independent variable $\alpha=\log a(t)$. Notice that $x^{2}$ and $y^{2}$ give the fraction of total energy density carried by the scalar field kinetic and potential energy, respectively. Then, we can rewrite the system (26-29) as

$$
\begin{aligned}
& x^{\prime}=-3 x+3 x\left[x^{2}+\frac{1}{2} w\left(1-x^{2}-y^{2}\right)\right]-\mu y^{2}+\frac{1}{2} \beta\left(1-x^{2}-y^{2}\right), \\
& y^{\prime}=\mu x y+3 y\left[x^{2}+\frac{1}{2} w\left(1-x^{2}-y^{2}\right)\right] .
\end{aligned}
$$

where the prime is here $d / d \alpha$. The system is invariant under the change of sign of $y$ and of $\alpha$. Since it is also limited by the condition $\rho^{*}>0$ to the circle $x^{2}+y^{2} \leq 1$, we may study only the unitary semicircle of positive $y$. The critical points, those that verify $x^{\prime}=y^{\prime}=0$, are scaling solutions, on which the scalar field equation of state is

$$
w_{\psi}=\frac{2 x^{2}}{x^{2}+y^{2}}=\text { const },
$$

the scalar field total energy density is $\Omega_{\psi}=x^{2}+y^{2}$, and the scale factor is

$$
a \sim t^{p}, \quad p=\frac{2}{3 w}\left[\frac{w}{w+\Omega_{\psi}\left(w_{\psi}-w\right)}\right]
$$

(the slope $p$ is not to be confused with the pressure).

Copeland et al. 10 have shown that the system (38) with $\beta=0$ and an exponential potential has up to five critical points, that can be classified according to the dominant energy density: one dominated by the scalar field total energy density (let us label this point as solution $a$ and refer to its coordinates as $x_{a}, y_{a}$ ), one in which the fractions of energy density in the matter and in the field are both non-zero (labelled $b$ ), one dominated by the matter field $(c)$, and finally two dominated by the kinetic energy of the scalar field, of which one at $x=-1(d)$ and one at $x=+1(e)$.

The critical points on which the matter field becomes negligible reduce to the $\beta=0$ case: therefore, the solutions $a, d$, and $e$ remain the same also for $\beta \neq 0$. The points $b$ and $c$ are instead modified. The solution $c$ is no longer matter dominated: rather, the scalar field kinetic energy and the matter energy take up a constant fraction of the total energy. In MDE, the scalar field kinetic energy amounts to $\Omega_{\psi}=1 / 9$. The critical points in the general case $\beta \neq 0$ are listed in Tab. I, where we put $g(\beta, w, \mu) \equiv \beta^{2}+2 \beta \mu+18 w$. 


\begin{tabular}{|c|c|c|c|c|c|}
\hline & $x$ & $y$ & $\Omega_{\psi}$ & $p$ & $w_{\psi}$ \\
\hline$a$ & $-\mu / 3$ & $\left(1-x_{a}^{2}\right)^{1 / 2}$ & 1 & $3 / \mu^{2}$ & $2 \mu^{2} / 9$ \\
\hline$b$ & $-\frac{3 w}{2 \mu+\beta}$ & $-x_{b}\left(\frac{g}{9 w^{2}}-1\right)^{1 / 2}$ & $\frac{g}{(\beta+2 \mu)^{2}}$ & $\frac{2}{3 w}\left(1+\frac{\beta}{2 \mu}\right)$ & $\frac{18 w^{2}}{g}$ \\
\hline$c$ & $\frac{\beta}{6-3 w}$ & 0 & $\left(\frac{\beta}{6-3 w}\right)^{2}$ & $\frac{6(2-w)}{\beta^{2}+9(2-w) w}$ & 2 \\
\hline$d$ & -1 & 0 & 1 & $1 / 3$ & 2 \\
\hline$e$ & +1 & 0 & 1 & $1 / 3$ & 2 \\
\hline \multicolumn{5}{|c|}{ Tab. I } \\
\hline
\end{tabular}

Although the number and position of the critical points is affected only quantitatively by the extra coupling, their stability properties are modified in a more radical way. In particular, while for $\beta=0$ only the points $a$ and $b$ can be attractors, here we show that also $c$ and $d$ may be stable. Only the point $e$ remains always unstable.

The stability analysis is performed as usual by linearization around the critical points. The parametric regions in which the real part of both eigenvalues of the linearization matrix is negative are regions of stability. To simplify the discussion, we only consider the crucial property of stability versus instability, paying no attention to the topography of the critical point (whether it is a knot, spiral, or saddle). In the following, we say that an attractor exists if it lies in the region $0 \leq x^{2}+y^{2} \leq 1$. The parameter spaces are plotted in Fig. 1.

Point $a$.

The solution $a$ exists for $\mu^{2}<9$, and is an attractor only for $\mu_{-}<\mu<\mu_{+}$where

$$
\mu_{ \pm}=\frac{1}{4}\left(-\beta \pm \sqrt{\beta^{2}+72 w}\right)
$$

(e.g., $\mu_{-}=-2.39$ and $\mu_{+}=1.89$ for $w=1$ ). On this attractor we have $w_{\psi}=2 \mu^{2} / 9$ and

$$
p_{a}=3 / \mu^{2}
$$

inflationary if $|\mu|<\sqrt{3}$.

Point $b$.

The attractor $b$ exists and is stable in the region delimited by $\mu<\mu_{-}$and $\mu>\mu_{+}$and the two branches of the curve

$$
\mu_{0}=-\frac{1}{2 \beta}\left(\beta^{2}+18 w-9 w^{2}\right)
$$

The scale factor slope on the attractor is

$$
p_{b}=\frac{2}{3 w}\left(1+\frac{\beta}{2 \mu}\right) .
$$

and, for $\beta=4-3 w$, is inflationary within the two branches of the curve

$$
\mu_{i}=\frac{4-3 w}{3 w-2}
$$

It is remarkable that the inflationary region for the point $b$ includes values smaller than $w \approx 0.91$, and therefore excludes the MDE equation of state $w=1$. This conclusion is not changed by replacing $\mu$ and $\beta$ with their counterparts $\mu_{q}$ and $\beta_{q}$ in the case of a quadratic coupling $f(\phi)$.

Point $c$.

This point exists for $w<5 / 3$, and is stable below the lower branch and above the upper branch of $\mu_{0}$. The slope is

$$
p_{c}=\frac{6(2-w)}{\beta^{2}+9(2-w) w},
$$

and it is never accelerated if $\beta=4-3 w$. The point $c$ shares with $b$ the property that matter and scalar field have both a non-vanishing fraction of the energy density.

Point $d$.

This point exists for all values of the parameters, and if $\beta=4-3 w$ is stable for $w>5 / 3$ and $\mu>3$. Its slope is always $p_{d}=1 / 3$.

Point $e$.

This point exists and is unstable for all values of the parameters if $\beta=4-3 w$. 
The complex structure of the parameter space is summarized in Fig. 2. Notice that 1), for each value of the parameters $w, \mu$ there is one and only one attractor; 2 ) for $w=1$ the points $a, b$ or $c$ can be stable, depending on $\mu$; 3) these solutions are inflationary in the shaded region; 4) only the point $a$ can be accelerated for $w=1$ or larger. In Fig. 3 we present four phase spaces displaying in turn the four possible attractors. The parameters correspond to the points marked with stars in Fig. 2. As already remarked, attractors $c$ and $d$ have not been previously noticed. Also, it is important to remark that the attractors are not only locally stable, but extend their basin of attraction to all of the phase space. That is, any possible initial condition lead to the attractor.

\section{BACK TO THE JORDAN FRAME}

Here we leave the dynamical analysis of the system in the rescaled frame and get back to the original one. What the attractors look like in the Jordan frame?

Reintroducing the tildes, we have along the attractors $a$ and $b$ (from now on, quantities without tildes are expressed in the original metric)

$$
c \psi=-\frac{2}{\mu} \log \left|\widetilde{t} / \tau_{a, b}\right|,
$$

(for $\mu \neq 0$ ) where

$$
\tau_{a, b}^{-1}=\sqrt{2 A} \mu c \frac{x_{a, b}}{y_{a, b}} .
$$

On the attractors $c$ and $d$, for which $y=0, \psi \rightarrow \infty$, and the conformal transformation cannot be performed. Since $\psi$ is proportional to $\log f$, the attractors $c$ and $d$ lead to an effective gravitational constant that is either zero or infinite and are therefore to be rejected as possible solutions in the Jordan frame. Of course, trajectories that have not already reached the attractor cannot be excluded, but these are not scaling solutions, and will not be further considered in this paper.

Form Eq. (46) it follows (neglecting the subscripts)

$$
e^{2 \omega}=(\tilde{t} / \tau)^{-2 / \mu}
$$

From the latter expression we can evaluate the relation between the old and new time and scale factor, given by Eq. (22) and (23). We obtain (for $\mu \neq 0,-1$ )

$$
\begin{aligned}
\tilde{t} & \sim t^{\frac{\mu}{1+\mu}}, \\
\widetilde{a} & \sim t^{-1 /(1+\mu)} a(t) .
\end{aligned}
$$

As can be seen, for $\mu \rightarrow \pm \infty$ the old and new metric coincide; in this limit the scalar field vanishes on the attractor, and the system reduces to the pure perfect fluid Friedmann case.

It follows that in the original variables the scale factor is again a power law

$$
a \sim t^{p^{\prime}}, \quad p^{\prime}=\frac{1+\mu p}{1+\mu} .
$$

On the attractor $a, p_{a}=3 / \mu^{2}$, which is inflationary (both in the original and in the rescaled frame) if $\mu^{2}<3$, that is $2-\sqrt{3}<M<2+\sqrt{3}$, we have

$$
p_{a}^{\prime}=\frac{\mu+3}{\mu(1+\mu)} .
$$

Consider now some special cases. If $0>\mu>-1$ the scale factor follows a pole-like inflation, $a \sim\left(t_{0}-t\right)^{p_{a}^{\prime}}$ with negative exponent. For $\mu=0$,(i.e. $V \sim f^{2}$ ) the old and new metric coincide (up to a constant), the field freezes to a constant and its energy drives a deSitter expansion. The system reduces asymptotically to pure general relativity with a cosmological constant. Finally, for $\mu=-1$ (i.e. $V \sim f$ ), the scale factor is power law accelerated in the new frame, but maps again to a deSitter expansion in the original frame. If $f$ is quadratic in $\phi$, then the inflationary condition on the solution $a$ reads

$$
\mu^{2}<3(1+1 / 6 \xi)
$$

On the attractor $b$, on the other hand, putting $p_{b}=2 /\left(3 w^{\prime}\right)$ with $w^{\prime}=w(1+\beta / 2 \mu)^{-1}$ we obtain 


$$
p_{b}^{\prime}=\frac{3 w^{\prime}+2 \mu}{3 w^{\prime}(1+\mu)}
$$

Notice that $p_{b}^{\prime} \rightarrow 2 / 3 w$ for $\mu \rightarrow \pm \infty$, as expected. Since the property of being accelerated is conformally invariant (for positive definite conformal factors), going back to the old frame do not change qualitatively the attractors found so far. Also, it is not difficult to check that $\Omega_{\rho^{*}}=\kappa^{2} \rho^{*} / 3 \widetilde{H}^{2}$ and $\Omega_{\psi}$ are invariant under conformal transformation, so that $\Omega_{\psi}=\Omega_{\phi}$.

It can be shown that the choice $V \sim f^{M}$ is the only one that allows scaling attractors in both the old and the new metric. Other choices are possible that allow scaling solutions either in the old or in the new metric: for instance, $V \sim f^{2}(\log f)^{M}$ gives scaling attractors in the new metric but not in the old one.

\section{COSMOLOGICAL PROPERTIES}

Once we have the analytical expression of the attractors, we must consider whether they are viable as cosmological solutions. The attractor solution $a$ is inflationary (accelerated) and the scalar field is asymptotically the dominating component. As such, it may match the observations of an accelerated expansion; for instance, the value $w_{\psi} \approx 0.4$ suggested in Ref. [26] implies

$$
M \approx 0.7 \text { or } 3.3 .
$$

On the other hand, since $\Omega_{\phi} \rightarrow 1$, in order to allow for a substantial fraction in the ordinary matter component at the present, the attractor has not to be already reached.

The solution $b$ has some drawbacks. First, is not accelerated at all for $w=1$; second, the constraints from nucleosynthesis do not allow a large fraction of energy density in the scalar field, so that it cannot provide closure energy. However, as argued in [2], models which reach this attractor compare favorably with observations of large scale structure, and may have a simple interpretation in terms of fundamental physics.

Both solutions $a$ and $b$ are heavily constrained by the upper limits on the variability of the gravitational constant. We have

$$
|\dot{G} / G|=|\dot{f} / f|=\frac{2}{|1+\mu|} \frac{1}{t}
$$

Comparing with the observational constraint $|\dot{G} / G|<a 10^{-10} \mathrm{yr}^{-1}$, and assuming $t \approx 10$ Gyr, we obtain the condition

$$
\mu>\frac{2}{a}-1 .
$$

Current constraints (see e.g. [27] ) give $a \approx 0.1$ or smaller. This implies $\mu>20$, too large for the attractor $a$ to exist. A similar problem arises if $f$ is quadratic. Along the attractor $b$, the energy density in the scalar field is a constant fraction of the total energy. In MDE (and for $\beta=4-3 w=1$ ) this is

$$
\Omega_{\phi}=\frac{19+2 \mu}{(1+2 \mu)^{2}} .
$$

The constraint (55) gives

$$
\Omega_{\phi} \leq 0.035,
$$

which confines the scalar field contribution to that of a minor component. This constraint is three or four times stronger than that imposed by the nucleosynthesis [2] on a minimally coupled field.

\section{CONCLUSIONS}

In this paper we have investigated a large class of NMC models in the limit of strong coupling with a perfect fluid matter component, searching for attractors that might provide a decaying cosmological constant. These models include all the cases in which the potential $V(\phi)$ is a power of the coupling function $f(\phi)$, regardless of their functional form. We have shown that

1. The NMC system can be reduced to a scalar field with an exponential potential, a minimal coupling to gravity, and an extra coupling to the matter. 
2. For each pair of the parameters $w, \mu$ there is one out of four possible scaling attractors: one, $a$, scalar field dominated and possibly accelerated; one, $b$, decelerated if $w \geq 0.91$ and with constant ratio of scalar field total energy to matter; one, $c$, always decelerated and with constant ratio of scalar field kinetic energy to matter; and finally one, $d$, also always decelerated, and dominated by the field kinetic energy.

3. Attractors $c$ and $d$ are acceptable only in the rescaled frame; in the original frame they lead to a gravitational constant either vanishing or infinite.

4. This choice $V \sim f^{M}$ is the only choice (in the strong coupling regime) for which there is a scaling attractor both in the original and in the rescaled metric.

5. The constraint on the time variability of $G$ rules out the accelerated models, and only allows a very small fraction of the energy density to be in the NMC scalar field.

Clearly, this analysis is not yet conclusive. Viable solutions might exist for which one or more of the following is true: $a$ ) the attractors are not yet reached; b) $V$ does not equal $f^{M} ; c$ ) the strong coupling regime does not apply. For instance, assuming $f=1+\kappa^{2} \xi \phi^{2}$, and in the limit of weak coupling, the $|\dot{G} / G|$

bound can be satisfied for small $\xi$, and the solutions are cosmologically acceptable, although by construction do not add much to the minimally coupled model.

\section{ACKNOWLEDGMENTS}

I am indebted to Carlo Baccigalupi, Francesca Perrotta and Jean-Philippe Uzan for useful discussions on the topic.

[1] J. Frieman, C. T. Hill, A. Stebbins, \& I. Waga, Phys. Rev. Lett. 75, 2077 (1995)

[2] P. G. Ferreira \& M. Joyce, Phys. Rev. D58, 2350 (1998)

[3] C. Wetterich, Nucl. Phys. B252, 668 (1985)

[4] I. Zlatev, L. Wang \& P. J. Steinhardt, astro-ph/9807002 (1998)

[5] R.R. Caldwell, R. Dave, \& P.J. Steinhardt, Phys. Rev. Lett. 80, 1582 (1998)

[6] K. Coble, S. Dodelson, J. Frieman, Phys. Rev. D55, 1851 (1997)

[7] F. Perrotta \& C. Baccigalupi, astro-ph/9811156

[8] P. Viana \& A. Liddle, Phys. Rev. D57, 674 (1988)

[9] A.R. Liddle \& R.J. Scherrer, astro-ph/9809272

[10] E. J. Copeland, A.R. Liddle \& D. Wands, Phys. Rev. D57, 4686 (1997)

[11] S. Perlmutter et al. Nature 391, 51 (1998)

[12] A. G. Riess et al. astro-ph/9805201 (1998)

[13] L. Amendola, Phys. Lett. B301, 175 (1993)

[14] F.S. Accetta, D.J. Zoller, M.S. Turner, Phys. Rev. D31, 3046 (1985)

[15] L. Amendola, M. Litterio \& F. Occhionero, J. Mod. Phys. A5, 3861 (1990)

[16] T. Futamase \& K. Maeda, Phys. Rev. D39, 399 (1989)

[17] U. Kasper, Nuovo Cim. B103, 291 (1989)

[18] J.-P. Uzan, UGVA-DPT 1998/12-1022

[19] P.J. Steinhardt, F. S. Accetta, Phys. Rev. Lett. 64, 2740 (1990)

[20] L. Amendola, D. Bellisai \& F. Occhionero, Phys. Rev. D47, 4267 (1993)

[21] C. Wetterich, A\&A, 301, 321 (1995)

[22] B. Ratra, \& P.J.E. Peebles, Phys. Rev. D37, 3406 (1988)

[23] J.D. Barrow \& S. Cotsakis, Phys. Lett. B214, 515 (1988)

[24] H.J. Schmidt, Class. Quantum Grav. 7, 1023 (1990)

[25] K.I. Maeda, Phys. Rev. D39, 3159 (1989)

[26] M.S. Turner \& M. White, Phys. Rev. D56, R4439 (1997)

[27] D.B. Guenther, L.M. Krauss \& P. Demarque, Ap.J., 498, 871 (1998) 


\section{FIGURE CAPTION}

Fig. 1.

Regions of existence and stability in the parameter space $w, \mu$. In all panels, the eigenvalues of the linearization matrix change sign across the thick lines. The color code is as follows: white regions indicate that the critical point does not exists; light gray regions, the point is unstable; dark gray regions, the point is stable. The dotted lines are at $w=4 / 3$ and $w=5 / 3$ and are useful landmarks in the parameter space. From top to bottom, parameter spaces of the critical points $a, b, c$ and $d$.

Fig. 2.

Regions of stability in the parameter spaces. Each region is labelled by the critical point that is stable in that region. The gray area indicates where the attractor is accelerated. The stars mark the values of the parameters for which we display in the next plot the phase space.

Fig. 3.

Phase spaces for various values of the parameters, corresponding to the points marked as stars in Fig. 2. The phase space is contained in the positive unitary semicircle. While the phase space of attractors $a$ and $b$ are qualitatively similar to those displayed in Copeland et al. 10, the phase space of attractors $c$ and $d$ have no counterpart for $\beta=0$. 

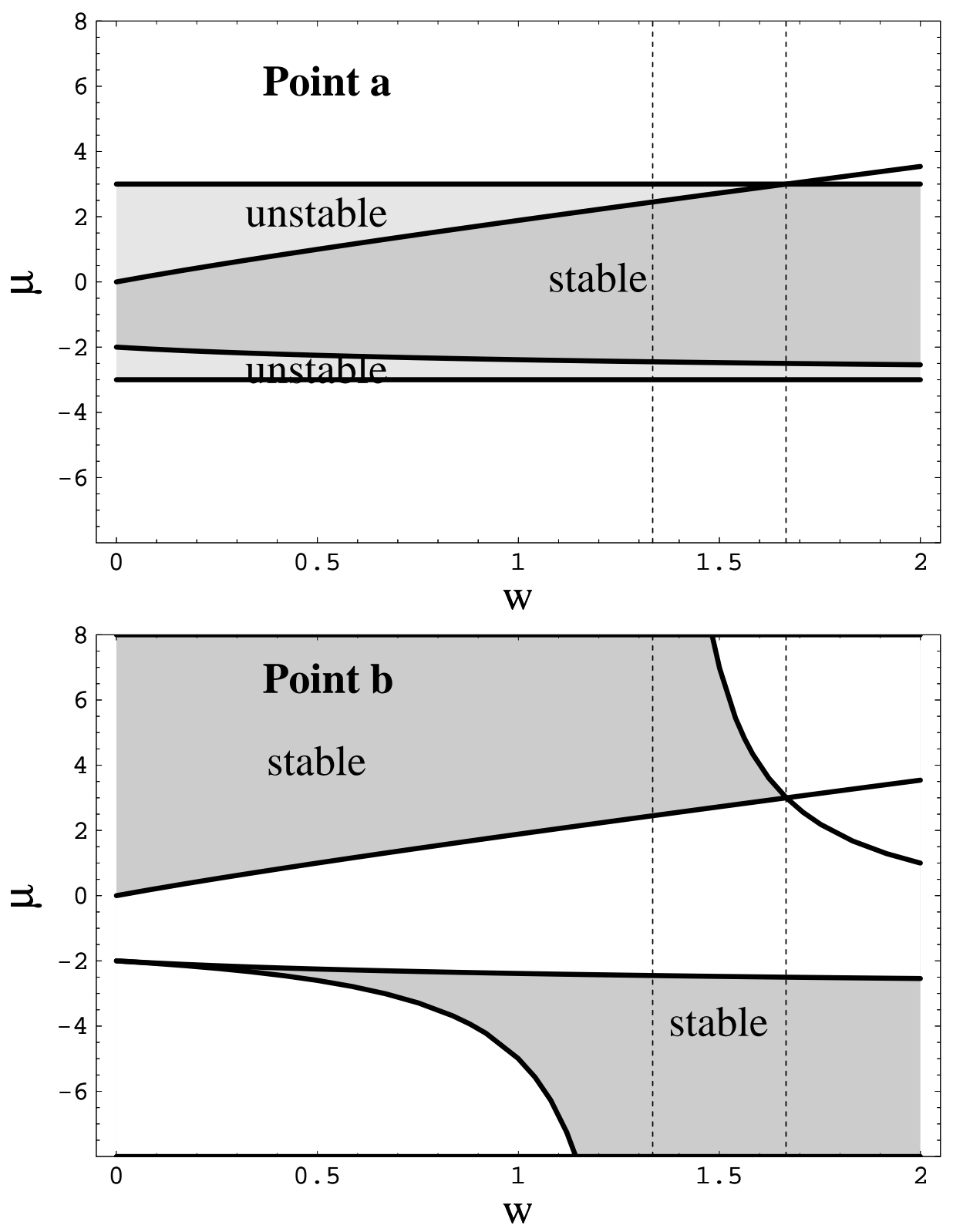

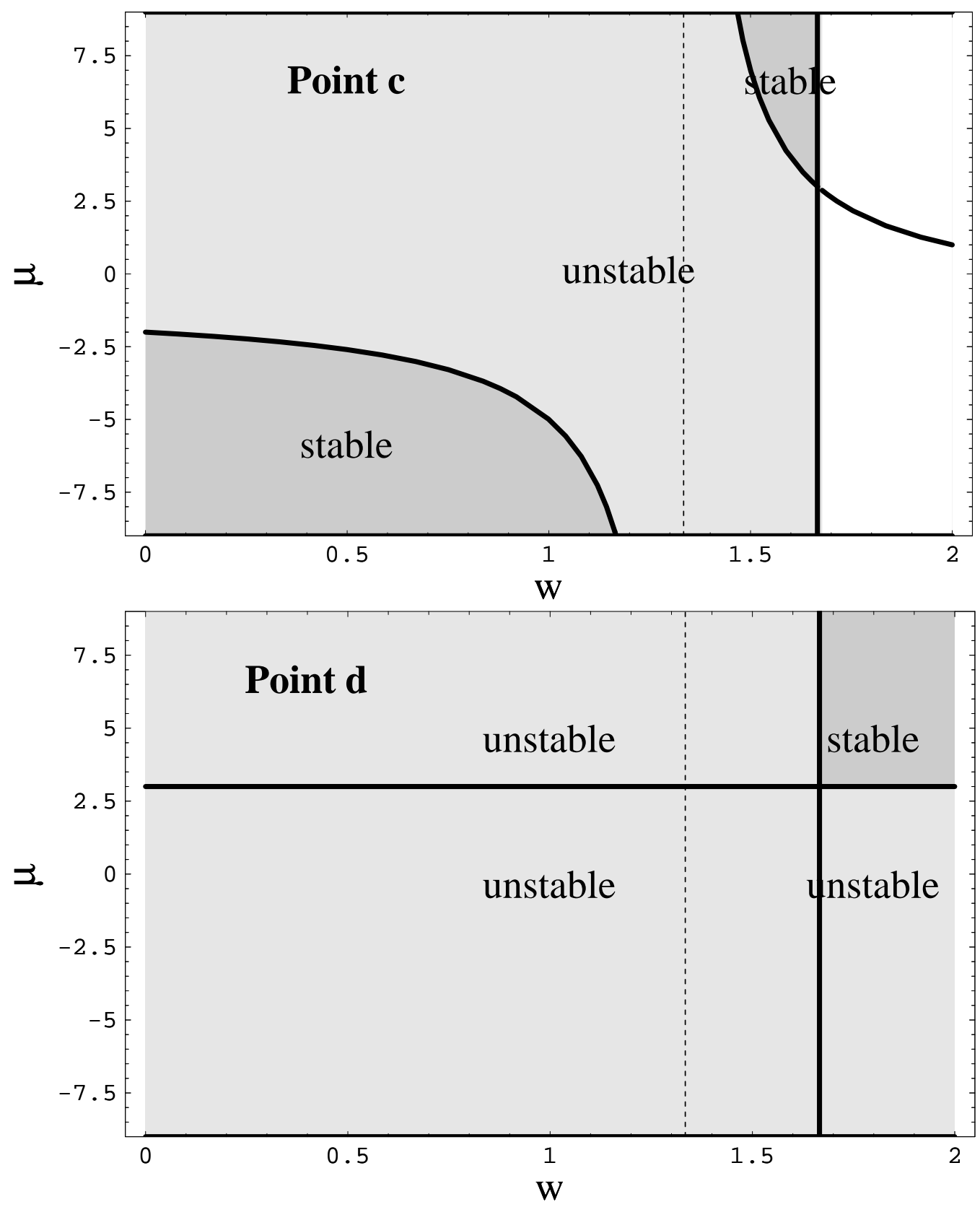


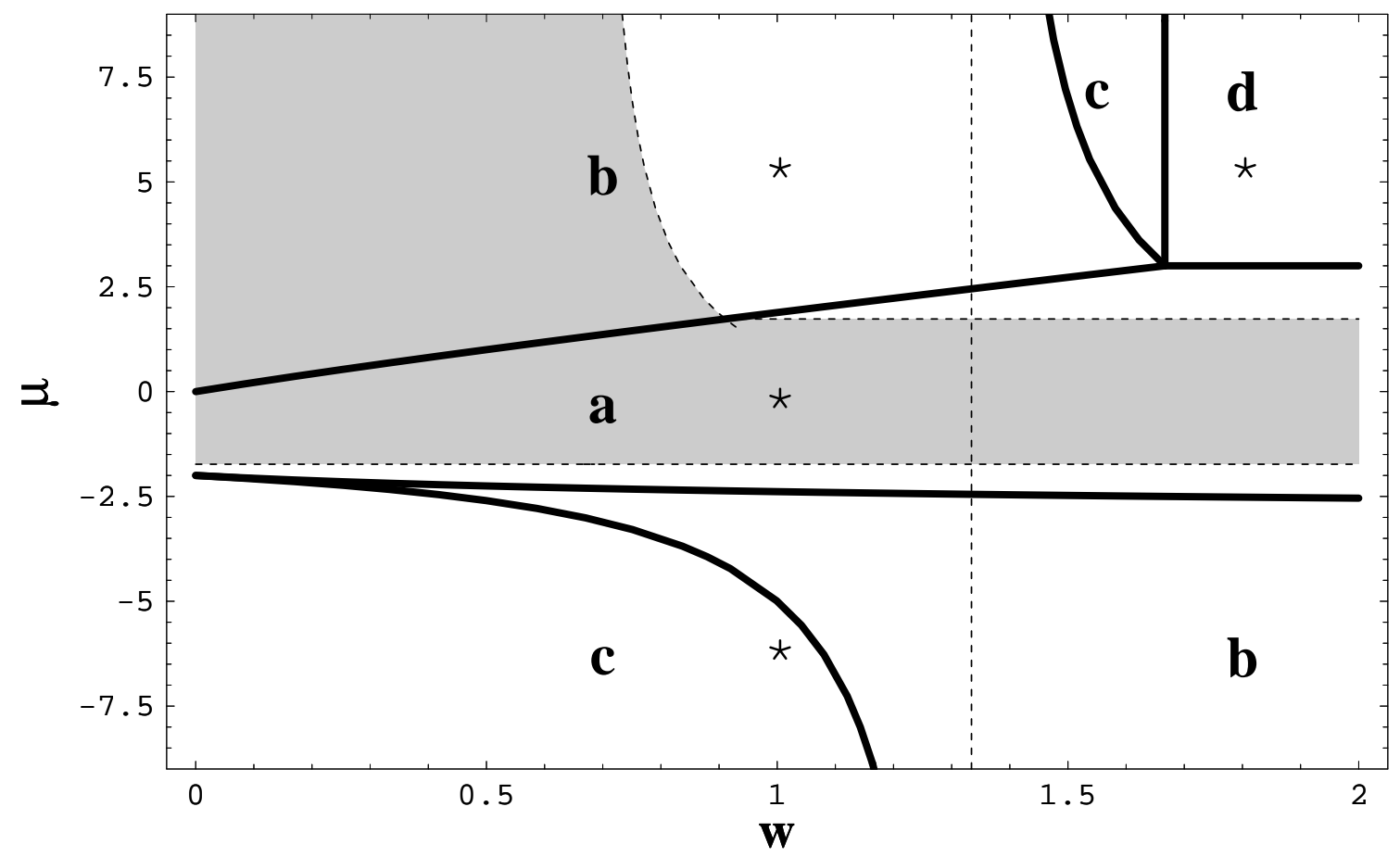



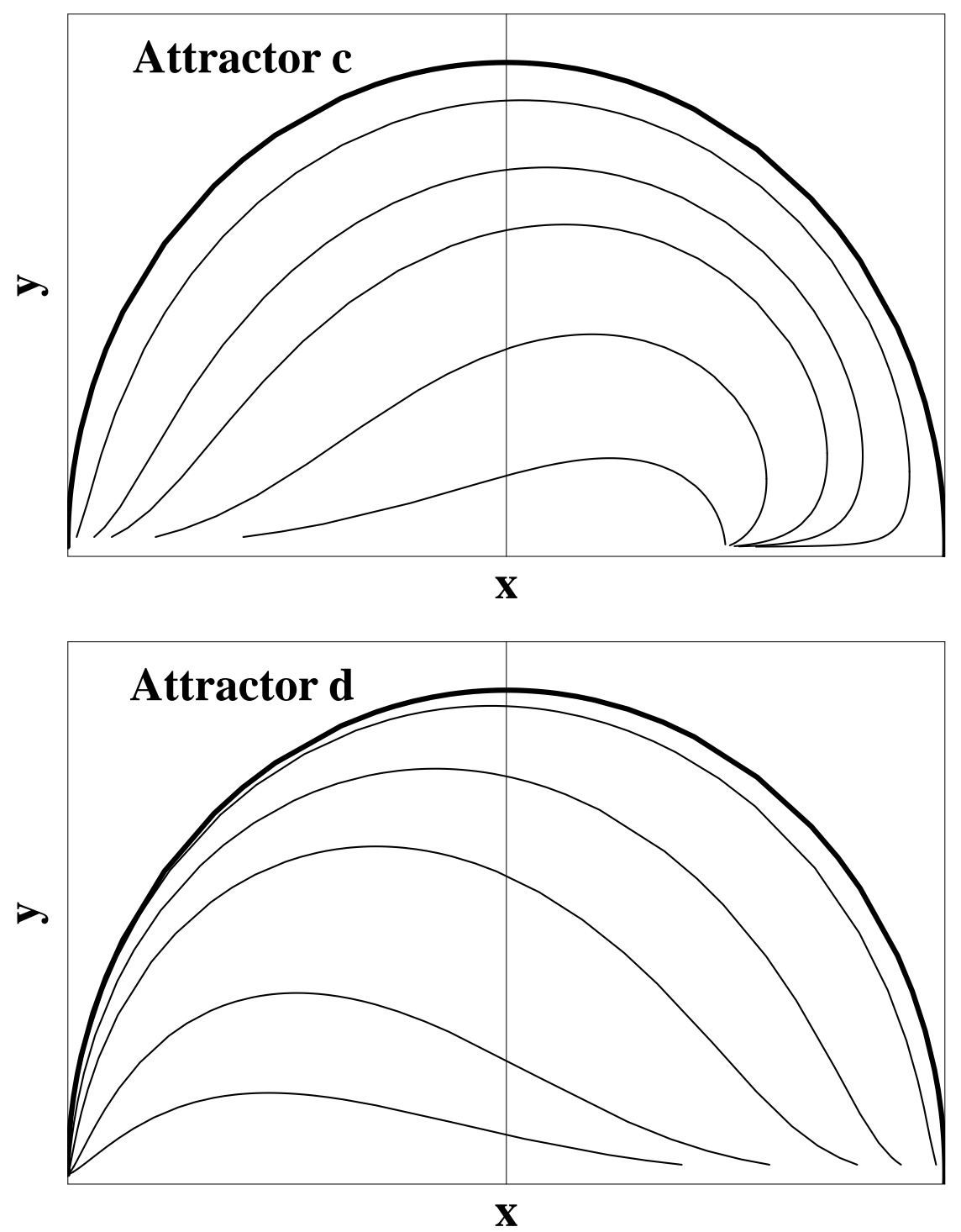

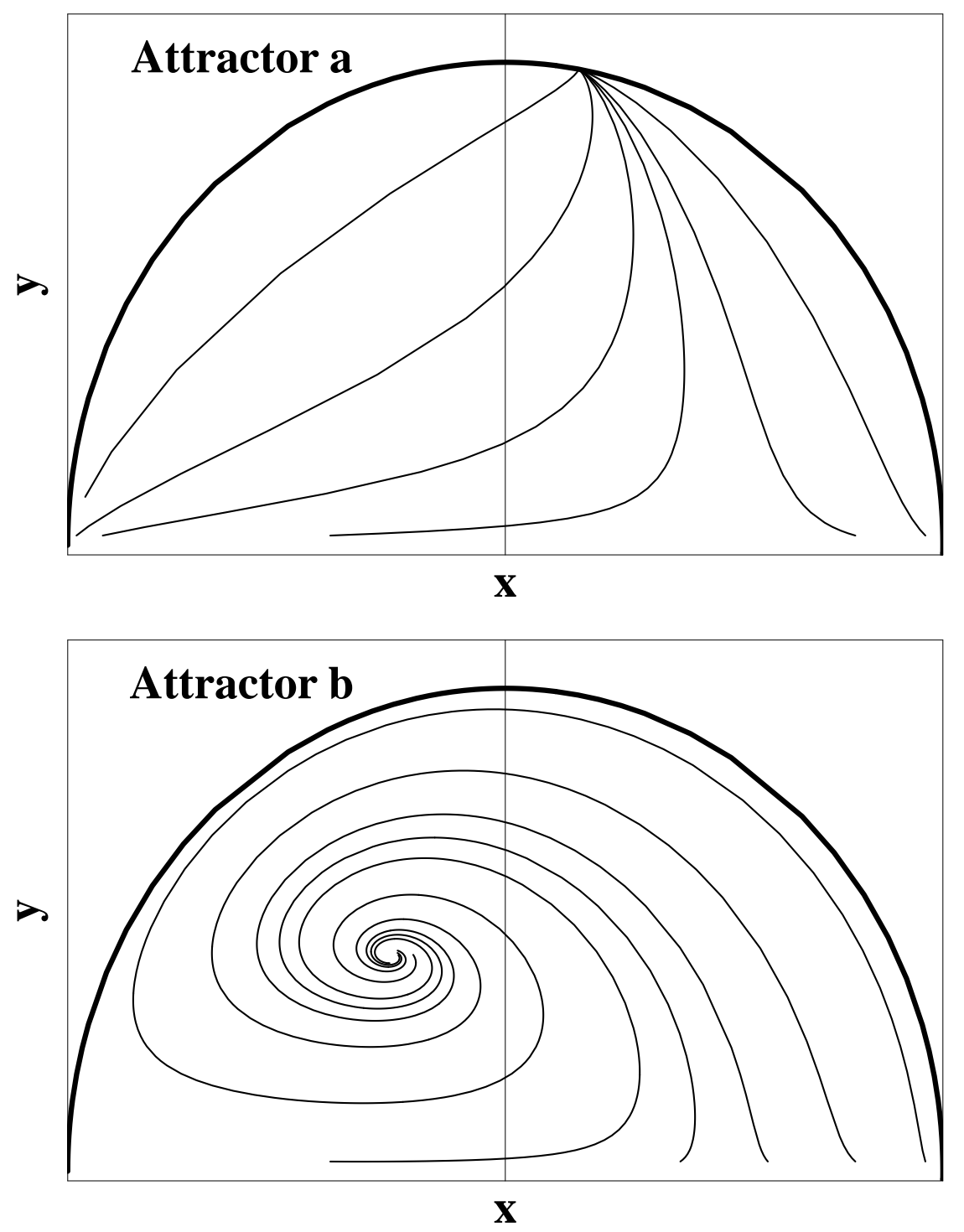